\title{
Un retard qui a du bon
}

\section{Yvonne Gilli}

Dre méd., membre du Comité central de la FMH, responsable du département Numérisation/eHealth

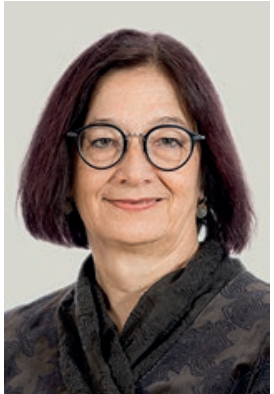

Le dossier électronique du patient (DEP) se trouve-t-il dans une impasse avant même son lancement? Mi-juillet, les médias nous apprenaient que la société Axsana AG avait facturé des montants de l'ordre de plusieurs dizaines voire centaines de milliers de francs aux hôpitaux qui lui sont affiliés, alors que le produit qu'elle propose ne remplit pas encore toutes les exigences de la loi. D'après le quotidien suisse alémanique Der Bund, la société serait bientôt à court d'argent [1]. Le DEP devrait fonctionner dans toute la Suisse depuis avril, mais son introduction a pris du retard, en particulier en raison de la complexité de la procédure de certification des communautés (de référence) chargées

\section{La FMH a mis sur pied un groupe de travail} chargé d'analyser plus en détail les aspects pratiques de la mise en œuvre du DEP.

d'exploiter le DEP. En dépit de la récente couverture médiatique, eHealth Suisse considère que la première mouture du DEP verra le jour au printemps 2021 [2].

Mais même si le DEP est effectivement introduit sur tout le territoire en 2021, la question de son utilité au quotidien pour les médecins reste ouverte. Pour analyser plus en détail les aspects pratiques de sa mise en œuvre, la FMH a récemment mis sur pied un groupe de travail.

Selon la loi, le DEP est un «dossier virtuel permettant de rendre accessibles [...] des données pertinentes pour [le] traitement qui sont tirées du dossier médical d'un patient et enregistrées de manière décentralisée». Ce n'est donc pas un outil de communication pour les médecins. Dans un premier temps, il ne devrait pas être possible d'y enregistrer des données structurées, ou tout au plus quelques-unes. Or les données structurées sont essentielles pour pouvoir trouver des informations ciblées et intégrer le dossier dans les systèmes primaires des cliniques et des cabinets. Deux conditions primordiales pour le travail quotidien des médecins, que ce soit du point de vue qualitatif ou économique (efficience).

Au-delà des idéaux d'exhaustivité des architectes du DEP, pour qui la priorité est de documenter les dossiers médicaux dans leurs moindres détails, les médecins auraient cependant besoin de pouvoir accéder le plus rapidement possible à certaines données critiques (cri- tical data set), dont les diagnostics et les médicaments actuels. Nous appelons donc ici les personnes concernées à tout faire pour que ces quelques données essentielles soient accessibles rapidement et de manière structurée.

Avec le DEP, la décision d'accorder les droits d'accès ou de supprimer certains documents revient aux patients. Pour nous, il ne s'agit en aucun cas de remettre en question ce principe d'autodétermination en phase avec la société actuelle. Néanmoins, il est légitime de se demander dans quelle mesure le DEP constitue un outil de communication si, faute d'un système adapté, les médecins doivent continuer de s'échanger des informations essentielles par courrier, fax ou e-mail, de crainte que le médecin en contact direct avec le patient n'ait pas accès au DEP alors que ce dernier lui aurait volontiers autorisé l'accès. D'autres pays comme le Danemark ou l'Estonie ont pallié ce problème en accordant un droit d'accès généralisé au DEP [3]. Pour protéger les données, tout accès est dûment consigné et les abus poursuivis pénalement. Dans ces pays aussi, l'autodétermination des patients est garantie grâce à la possibilité de limiter l'accès à certains documents.

Aujourd'hui, la plupart des cabinets médicaux ont franchi le pas du numérique et les médecins sont prêts à investir dans un système informatique efficace. S'ils participent au DEP, ils sont aussi tenus d'y recourir de manière adaptée, ce qui implique un investissement supplémentaire qui n'est pas pris en compte dans la

\section{Le retard actuel représente une occasion idéale} pour poursuivre le développement du DEP en tenant compte des besoins des médecins.

structure tarifaire. Même après l'introduction du DEP, ils devront continuer de consacrer une partie de leur temps à l'étude du dossier (hors DEP) et à la gestion de leur système informatique. Dans sa conception actuelle, le DEP nécessite en effet une double comptabilité, en particulier en l'absence de connexion entre le DEP et le dossier informatisé.

Le retard pris actuellement par l'introduction du DEP représente donc une occasion idéale pour poursuivre son développement en tenant compte des besoins des médecins. 